> L'avènement des anticorps monoclonaux (Acm) thérapeutiques a révolutionné le traitement de nombreuses affections, en particulier cancéreuses. Le ciblage de l'angiogenèse tumorale par les Acm occupe dorénavant une place privilégiée dans l'approche thérapeutique de ces maladies. Ce processus multifactoriel fait intervenir de nombreuses molécules synthétisées par la cellule tumorale ou son microenvironnement, certaines pro-angiogéniques, d'autres anti-angiogéniques. Le grand nombre de produits actuellement à l'étude ciblant, entre autres, la voie du vascular endothelial growth factor (VEGF), du plateletderived growth factor (PDGF) et des intégrines, reflète cette complexité. Le bévacizumab est actuellement le seul Acm utilisé en pratique clinique ciblant directement la néo-angiogenèse tumorale. II a prouvé son efficacité dans le traitement de nombreux cancers, et ce succès ouvre la voie au développement d'autres Acm ciblant des voies différentes de régulation de la vascularisation tumorale. Au-delà de la cancérologie, le ciblage vasculaire par les Acm suscite un intérêt marqué dans d'autres domaines de la médecine. II pourrait constituer un traitement de choix dans certaines pathologies cardiovasculaires et rhumatologiques et constitue désormais un standard du traitement de certaines affections ophtalmologiques. <

\section{Anticorps \\ monoclonaux \\ thérapeutiques \\ et ciblage \\ vasculaire}

Nicolas Cézé, Thierry Lecomte, Hervé Watier

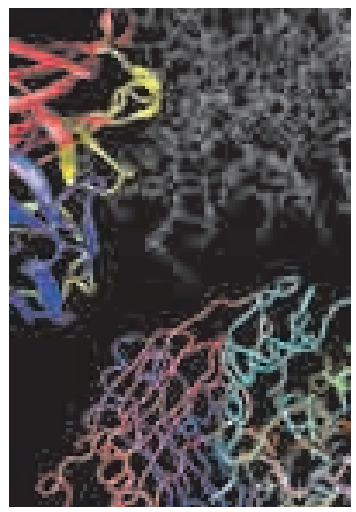

N. Cézé : CHRU de Tours, service d'hépato-gastroentérologie et d'oncologie digestive (NC, TL) et Laboratoire d'immunologie (HW) ; Université François-Rabelais de Tours ; Service d'hépatogastroentérologie et d'oncologie digestive, CHRU de Tours, Hôpital Trousseau, 37044 Tours Cedex, France.

cezenicolas@yahoo.fr T. Lecomte, H. Watier : CHRU de Tours, service d'hépatogastroentérologie et d'oncologie

tumoral dont la croissance (tumorigenèse) est conditionnée par les interactions avec son microenvironnement, en particulier l'angiodigestive (NC, TL) et Laboratoire d'immunologie (HW) ; Université François-Rabelais de Tours; CNRS, UMR 6239 génétique, immunothérapie, chimie et cancer, Tours, France.

L'angiogenèse, à l'origine du développement et du renouvellement des capillaires, est un processus physiologique qui se produit lors de l'embryogenèse et des phases de croissance et de réparation tissulaires. En 1971, Judah Folkman fut le premier à évoquer son rôle dans la croissance des tumeurs [1]. Depuis, de nombreux travaux ont permis de mieux appréhender l'ensemble des processus complexes qui gouvernent l'angiogenèse normale et pathologique.

Une tumeur maligne évolue d'une phase préinvasive vers un phénotype agressif et métastatique à la suite de l'apparition de néovaisseaux favorisant sa croissance et sa dissémination à distance. Cette évolution, dénommée «switch angiogénique », est sous la dépendance d'altérations de l'équilibre entre des facteurs pro- et anti-angiogéniques, sécrétés par la cellule tumorale ou son microenvironnement.

Les premiers essais d'inhibiteurs de l'angiogenèse (angiostatine, thrombospondine, protamine, etc.) datent des années 1980. Leur peu de succès était probablement imputable au manque de spécificité de 
l'activité de la molécule. L'émergence des Acm, de grande spécificité pour leur antigène, a permis de franchir cet obstacle en ciblant des facteurs moléculaires clés intervenant dans l'angiogenèse.

\section{Ciblage de la voie du vascular endothelial growth factor (VEGF) : l'expérience du bévacizumab}

\section{La famille du vascular endothelial growth factor et l'angiogenèse tumorale}

La famille du VEGF comporte plusieurs membres (VEGF-A, B, C, D et ह) [2] parmi lesquels le VEGF-A (ou simplement VEGF) est le mieux connu. Son niveau d'expression est contrôlé par la disponibilité de l'oxygène dans les tissus: I'hypoxie est un régulateur positif via la voie de l'hypoxia inducing factor (HIF) [3]. Le VEGF-A, surexprimé dans de nombreux modèles tumoraux $[4,5]$, peut lier trois types de récepteurs membranaires à activité tyrosine kinase. Le VEGFR-1 (Flt1) est surtout exprimé par les cellules endothéliales et intervient dans leur capacité de migration. Le VEGFR-2 (Flk-1) est également exprimé par les cellules endothéliales. Activé par sa liaison au VEGF, il induit une régulation positive de la survie, des capacités de migration et de prolifération des cellules endothéliales et une augmentation de la perméabilité vasculaire. Enfin, un troisième récepteur, le VEGFR-3 (Flt4 ), est capable de lier les autres membres de la famille du VEGF. II est exprimé par les cellules endothéliales des vaisseaux lymphatiques et son activation stimule la lymphangiogenèse [27].

Progrès thérapeutiques et mise au point du bévacizumab (Avastin ${ }^{\circledR}$ ) La voie du VEGF et de ses récepteurs, très étudiée, peut être la cible de nombreux médicaments: Acm thérapeutiques, inhibiteurs de tyrosine-kinases, oligonucléotides antisens, VEGF$\operatorname{TRAP}^{1}(\rightarrow)$. Les travaux ciblant cette voie ont notam-

$\rightarrow$ voir N. Leveziel et al., page 1105 ment abouti à la mise au point du bévacizumab, un anticorps IgGl antiVEGF humanisé dérivant de l'anticorps murin A.4.6.1, et devenu un standard du traitement de différents cancers (colorectal, rein, sein, poumon). Le bévacizumab (Avastin ${ }^{\circledR}$ ) est actuellement l'unique représentant des Acm anti-angiogéniques utilisables en clinique hors essai thérapeutique. C'est en 2004 que Hurwitz et al. ont rapporté les résultats d'une étude de phase III (environ 800 patients inclus), réalisée en double aveugle et contrôlée contre placebo, qui évaluait le bénéfice potentiel de l'association du bévacizumab à une chimiothérapie à base de fluoropyrimidines, leucovorine et irinotécan $^{2}$ chez des patients traités en première ligne pour un cancer colorectal métastatique [6]. Les résultats de cette étude étaient significativement en faveur du bras de traitement comprenant le bévacizumab en termes de survie globale $(20,3$ versus 15,6 mois, $p<0,001)$, de survie sans progression ( 10,6 versus 6,2 mois, $p<0,001)$ et de taux de réponses objectives $(44,8$ versus $34,8 \%, p<0,004)$. Même s'ils apparaissent à première vue assez minces, ces progrès ont soulevé chez les cliniciens oncologues un enthousiasme qui a rapidement fait du bévacizumab un standard du traitement de pre-

${ }^{1}$ Le VEGF-Trap est une protéine fusionnant une portion du VEGFRl humain et du domaine extracellulaire du VEGFR2 avec le fragment Fc des IgGl humaines.

${ }^{2}$ Inhibiteur de topoisomérase de type 1. mière ligne des cancers colorectaux métastatiques. En effet, jamais au cours de la dernière décennie de telles avancées n'avaient pu être obtenues dans le traitement d'une affection de pronostic aussi péjoratif. Néanmoins, cet enthousiasme a été tempéré fin mai dernier, lors du congrès de l'American society of clinical oncology (ASCO) au cours duquel ont été rapportés les résultats de l'essai C-08. Cet essai évaluait l'efficacité du bévacizumab administré pendant six mois en association à une chimiothérapie de type FOLFOX ${ }^{3}$ puis pendant six mois en monothérapie en situation adjuvante avec résection d'un cancer colorectal de stade II ou III. Ces résultats étaient décevants puisque l'association du bévacizumab à une chimiothérapie par FOLFOX n'apportait pas de bénéfice sur la survie sans récidive à trois ans par rapport au FOLFOX seul.

Toutefois, les résultats encourageants obtenus avec le bévacizumab dans le cancer colorectal métastatique ont été reproduits dans plusieurs autres types de cancer. $\varepsilon$ n 2006, Sandler et al. [7] rapportaient les résultats d'un essai évaluant le bénéfice de l'association du bévacizumab à une chimiothérapie à base de taxanes et de sels de platine chez des patients traités pour un carcinome pulmonaire non à petites cellules avancé ou métastatique (stades IIIb et IV). Les résultats faisaient état d'un bénéfice significatif lié à l'administration de bévacizumab en termes de survie globale $(12,3$ versus 10,3 mois, $p<0,003)$, de survie sans progression $(6,2$ versus 4,5 mois, $p<0,001)$ et de taux de réponses objectives ( $35 \%$ versus $15 \%, p<0,001$ ). Toutefois, ce bénéfice était obtenu au prix d'un surcroît de mortalité lié au traitement dans le bras recevant le bévacizumab, en particulier en raison de la survenue de plusieurs cas d'hémoptysies fatales. Par la suite, les équipes de Miller [8] en 2007 et de Rini [9] en 2008 ont fait état des résultats obtenus dans des essais de phase III évaluant le bénéfice de l'association du bévacizumab respectivement au paclitaxel ${ }^{4}$ dans le cancer du sein métastatique, et à l'interféron dans le cancer du rein avancé ou métastatique [28]. Dans ces deux essais, l'administration du bévacizumab apportait un bénéfice en termes de taux de réponses objectives et de survie sans progression. Depuis la publication de ces essais, le bévacizumab a obtenu des autorisations de mise sur le marché dans ces quatre indications (cancer du côlon métastatique en association à une chimiothérapie à base de 5 -fluorouracile, cancer du poumon non à petites cellules avancé ou métastatique en association aux sels de platine, cancer du sein métastatique en association au paclitaxel et cancer du rein avancé ou métastatique en association à l'interféron).

\footnotetext{
${ }^{3}$ Association de 5-fluorouracile, d'oxaliplatine et de leucovérine.

${ }^{4}$ Paclitaxel ou Taxo| ${ }^{\circledR}$ : alcaloïde dérivé de l'écorce d'ifs de la famille des taxanes et empêchant le désassemblage des microtubules.
} 
La tolérance du bévacizumab est globalement bonne chez la plupart des patients traités, les toxicités les plus fréquentes étant l'hypertension artérielle et la protéinurie, dont la prise en charge fait maintenant l'objet de recommandations [10]. De rares complications plus sévères, hémoptysies, perforations gastro-intestinales ou manifestations thrombo-emboliques, ont cependant été rapportées.

L'efficacité de l'inhibition de la voie du VEGF par le bévacizumab dans plusieurs types de cancer ouvre la voie à de nouvelles indications de ce médicament. L'autorisation de mise sur le marché pourrait prochainement être attribuée au bévacizumab dans les gliomes malins de stade III ou les glioblastomes, en association à l'irinotécan à la suite des travaux de Vredenburgh [11].

\section{Ciblage vasculaire par les Acm thérapeutiques: I'histoire ne fait que commencer}

\section{Nouveaux développements dans le ciblage du VEGF}

La success story du bévacizumab ouvre la voie au développement et à I'utilisation en clinique de nouveaux Acm anti angiogéniques qui sont actuellement évalués dans le cadre d'études cliniques. Grâce à l'expérience clinique acquise avec le bévacizumab, les thérapeutiques anti angiogéniques ciblant la néo angiogenèse tumorale, et en particulier la voie du VEGF, représentent l'une des armes de choix de la stratégie anticancéreuse. L'AMG-706 et le HuMV833, autres Acm anti-VEGF, font l'objet d'études précliniques (Tableau I).

D'autres Acm, ciblant les récepteurs du VEGF, sont à l'essai, tels que I'IMC- $1 C l 1^{5}$, un anticorps chimérique, et le $D C 101$, tous deux dirigés contre le VEGFR-2 [12]. L'IMC-V18 est un autre Acm humanisé, spécifique du VEGFR-1. D'autres sont développés sous la forme de fragments Fab, comme I'IMC-1121B, humanisé et spécifique du VEGFR-2 [13] et le CDP-791, Fab pégylé humanisé anti-VEGFR-2.

\section{Autres cibles pro-angiogéniques potentielles \\ Le ciblage du système VEGF-VEGFR n'est que l'une des multiples appro- ches qu'offre la complexité des mécanismes de l'angiogenèse pour envisager la mise au point de nouvelles thérapeutiques anti-angio- géniques dans le traitement du cancer. De très nombreuses autres cibles thérapeutiques potentielles font l'objet d'études plus ou moins abouties (Tableau I).}

\section{Le platelet-derived growth factor (PDGF)}

Ce facteur de croissance appartient à la même famille que le VEGF. Sécrété par les cellules endothéliales, il est surexprimé au cours de nombreux cancers (poumon, prostate, etc.). Il se lie à deux récepteurs, PDGFR $\alpha$ et PDGFR $\beta$, entraîne la prolifération des péricytes (cellules musculaires périvasculaires nécessaires à la stabilisation et à la maturité des vaisseaux) voisins et des cellules musculaires lisses, et stimule leur migration et leur assemblage au sein de la paroi vasculaire [17]. L'inhibition de l'interaction PDGF-PDGFR a fait l'objet de travaux chez I'animal. L'Acm 1B3, anti-PDGFR $\beta$, a montré son efficacité dans des

${ }^{5}$ IMC : dénomination des Acm produits par les laboratoires ImClone Systems Incorporated. modèles murins de cancers pancréatiques et pulmonaires [18]. L'AMT 3G3, anti-PDGFR $\alpha$ entièrement humain, est en phase d'essais précliniques.

\section{La famille des fibroblast growth factors (FGF)}

Elle comporte plus de vingt membres, FGFl et FGF2 étant les mieux connus [19]. Ces facteurs de croissance lient des récepteurs (FGFR 1 à 4) exprimés à la membrane des cellules endothéliales. La liaison FGF-FGFR aboutit à une stimulation autocrine de la prolifération et de la migration des cellules endothéliales au moins égale in vitro à celle qu'induit le VEGF. La sécrétion de FGF intervient également dans la morphogenèse des microcapillaires. Le FGF est surexprimé dans certains cancers (prostate, mélanome, etc.). Les altérations touchent aussi les récepteurs, sous la forme de mutations activatrices et de surexpressions, particulièrement celles de FGFR2, au cours de certains autres types de tumeur (vessie, sein, estomac). Le système FGF-FGFR est donc une cible thérapeutique d'intérêt, et plusieurs travaux ont démontré l'efficacité in vivo d'Acm anti-FGF. Le KM1334, Acm thérapeutique anti-FGF8b, s'est montré capable d'induire une réduction tumorale dans un modèle de carcinome mammaire murin [20]. Enfin, les IMC-Al, IMC-4H et IMC$\mathrm{H} 7$ sont trois $\mathrm{Acm}$ anti-FGFRI en cours de développement, entièrement humanisés et de sous-classe IgGl.

\section{Le transforming growth factor $\boldsymbol{\beta}$ (TGF $\boldsymbol{\beta}$ )}

Cet autre facteur de croissance pro angiogénique est synthétisé par les cellules tumorales ou leur microenvironnement. II est capable d'engager deux types de récepteurs (TGF $\beta-\mathrm{R}$ I et II). La surexpression du TGF- $\beta$ est fréquente dans certains cancers (prostate) auxquels il confère un phénotype agressif, en augmentant leur potentiel angiogénique et métastasiant [21]. La neutralisation du TGF- $\beta$ a fait l'objet de travaux préliminaires aux résultats encourageants. Un anticorps anti-TGF- $\beta$ a ainsi permis de réduire de façon significative la densité microvasculaire de tumeurs prostatiques in vitro [22].

\section{La famille des angiopoïétines}

Elle regroupe cinq membres (Ang-1, 2, 3, 4 et 5), ligands des récepteurs Tie2 qui sont sélectivement exprimés par les cellules endothéliales. Ang-1 et Ang-2, les mieux connus, sont antagonistes in vivo: l'interaction Ang1/Tie2 assure la survie des cellules endothéliales et la stabilité de leur intégration dans l'architecture de la paroi vasculaire. À l'inverse, l'interaction Ang-2/Tie2 entraîne leur prolifération et leur migration sous réserve de la présence de VEGF et de FGF dans le milieu. En l'absence de ces derniers, Ang-2 semble plutôt induire 


\begin{tabular}{|c|c|c|c|}
\hline $\begin{array}{l}\text { Facteurs } \\
\text { angiogéniques }\end{array}$ & Effets biologiques & Implication en cancérologie & $\begin{array}{l}\text { Anticorps } \\
\text { en développement }\end{array}$ \\
\hline VEGF-VEGFR & $\begin{array}{l}\text { VEGFR-1: (+) migration des cellules } \\
\text { endothéliales (CE) } \\
\text { VEGFR-2: (+) survie, capacité de } \\
\text { migration et prolifération } \\
\text { (+) Perméabilité vasculaire }\end{array}$ & $\begin{array}{l}\text { L'hypoxie induit l'expression du VEGF-A } \\
\text { [3] } \\
\text { Le VEGF-A est surexprimé dans de nom- } \\
\text { breux modèles tumoraux }[4,5]\end{array}$ & $\begin{array}{l}\text { AMG-706, HuMV833 } \\
\text { (anti-VEGF) } \\
\text { IMC-1C11, DC101 } \\
\text { (anti-VEGFR-2) [12] } \\
\text { IMC-V18 } \\
\text { (anti-VEGFR-1) }\end{array}$ \\
\hline $\begin{array}{l}\text { PDGF- } \\
\text { PDGFR }\end{array}$ & $\begin{array}{l}\text { (+) Prolifération des péricytes et des } \\
\text { cellules musculaires lisses } \\
\text { (+) Migration, assemblage dans la } \\
\text { paroi vasculaire }[17]\end{array}$ & $\begin{array}{l}\text { Surexpression du PDGF dans plusieurs } \\
\text { types de cancers (pancréas, poumon) }\end{array}$ & $\begin{array}{l}\text { lB3 [18] } \\
\text { (anti-PDGFR } \beta \text { ) } \\
3 G 3 \\
\text { (anti-PDGFR } \alpha \text { ) }\end{array}$ \\
\hline FGF-FGFR & $\begin{array}{l}\text { (+) Prolifération et migration des CE } \\
\text { (autocrines) [19] } \\
\text { (+) Morphogenèse des microcapillaires }\end{array}$ & $\begin{array}{l}\text { FGF : surexpression fréquente (prostate, } \\
\text { mélanome) } \\
\text { FGFR : surexpression et/ou mutations } \\
\text { activatrices (vessie, sein, estomac) }\end{array}$ & $\begin{array}{l}\text { KMI334 [20] } \\
\text { (anti-FGF8b) } \\
\text { IMC-Al, IMC-4H, } \\
\text { IMC-H7 } \\
\text { (anti-FGFRI) }\end{array}$ \\
\hline TGF $\beta$-TGF $\beta R$ & $\begin{array}{l}\text { (+) Angiogenèse } \\
\text { (+) Potentiel métastatique des } \\
\text { cellules tumorales }\end{array}$ & $\begin{array}{l}\text { Surexpression de TGF } \beta \text { (prostate) + } \\
\text { phénotype tumoral agressif [21] }\end{array}$ & Anti-TGF $\beta[22]$ \\
\hline $\begin{array}{l}\text { Angiopoïétines (Ang-1, } \\
\text { Ang-2) et leurs récepteurs }\end{array}$ & $\begin{array}{l}\text { Ang-1 et Ang-2 lient le récepteur Tie-2 } \\
\text { Ang-1 : (+) survie des CE, intégration } \\
\text { dans la paroi vasculaire } \\
\text { Ang-2: (+) prolifération et migration } \\
\text { des CE (si VEGF et FGF) [23] }\end{array}$ & $\begin{array}{l}\text { Tie-2 : sélectivement exprimé par les C } \varepsilon \\
\text { La neutralisation d'Ang-2 diminue la } \\
\text { densité microvasculaire tumorale }\end{array}$ & Anti-Ang-2 [24] \\
\hline Intégrines & $\begin{array}{l}\text { (+) Adhésion cellule-cellule } \\
(+) \text { Adhésion cellule-MEC }\end{array}$ & $\begin{array}{l}\text { (+) Migration et angiogenèse (via l'orga- } \\
\text { nisation du cytosquelette, la forme et la } \\
\text { motilité des cellules endothéliales) }\end{array}$ & $\begin{array}{l}\text { Anti- } \alpha v \beta 3 \\
\text { Étaracizumab (Abégrin }{ }^{\circledR} \text { ) } \\
\text { a été testé avec succès } \\
\text { en phase I, est actuel- } \\
\text { lement en phase II [25] }\end{array}$ \\
\hline
\end{tabular}

Tableau I. Les principales cibles moléculaires faisant l'objet d'essais en thérapeutique anti-angiogénique. (+) : effet de stimulation ; MEC : matrice extracellulaire; $\subset \varepsilon$ : cellules endothéliales.

la régression des structures vasculaires [23]. Des études préliminaires d’Acm thérapeutiques anti-Ang-2 ont été menées in vivo. Oliner et al. [24] ont montré que ces Acm pouvaient entraîner une diminution de la microvascularisation tumorale associée à une régression, voire une éradication, de la tumeur dans un modèle murin.

\section{Les intégrines}

Ces récepteurs hétérodimériques transmembranaires interviennent dans les phénomènes d'adhésion cellule-cellule et d'interaction cellule-matrice extracellulaire (MEC). Ce sont des dimères $\alpha \beta$. On compte à ce jour dix-huit chaînes $\alpha(110-140 \mathrm{kDa}$ ) et huit chaînes $\beta$ (85$91 \mathrm{kDa}$ ), se combinant en vingt-deux couples $\alpha \beta$ fonctionnels. L'extrémité amino-terminale de l'intégrine est extracellulaire et possède une fonction de récepteur liant des éléments de la MEC (fibronectine, collagènes, laminine, etc.). L'extrémité carboxy-terminale, intracel-
Iulaire, lie le cytosquelette et est capable d'activer plusieurs voies de transduction du signal. L'expression différentielle des différents dimères d'intégrines régule l'interaction cellules-MEC. Certains de ces dimères sont à l'origine d'une réorganisation du cytosquelette affectant la motilité et la forme des cellules endothéliales, et favorisant leur migration et l'angiogenèse. Le couple $\alpha v \beta 3$, récepteur de la fibronectine, est peu exprimé, sauf au niveau des cellules activées lors de l'angiogenèse. Expérimentalement, les souris invalidées pour $\alpha v \beta 3$ n'expriment aucune vasculogenèse, ce qui explique que les nombreux travaux préliminaires utilisant cette intégrine aient été rapidement couronnés de succès. Ces travaux ont abouti au développement d'Acm anti- $\alpha v \beta 3$ actuellement en cours d'essai chez l'homme. 
L'étaracizumab (Abégrin ${ }^{\circledR}$ ), anticorps humanisé anti- $\alpha v \beta 3$ a fait l'objet d'études de phase I chez des patients atteints de différents cancers métastatiques avec des résultats probants et il est actuellement testé en phase II [25]. D'autres couples d'intégrines interviennent dans l'angiogenèse tumorale et font l'objet de travaux préliminaires de ciblage par des Acm, comme le volociximab, IgG4 anti-intégrine $\alpha 5 \beta 1$.

\section{Marqueurs d'angiogenèse utilisés comme signaux d'adressage}

Le ciblage par un Acm d'une voie de signalisation cellulaire à l'origine de signaux de prolifération néovasculaire n'est pas toujours une stratégie univoque d'inhibition pure. Ainsi, la spécificité d'expression de certains marqueurs de l'angiogenèse tumorale, comme les intégrines $\alpha v \beta 3$, peut être exploitée comme un signal d'adressage pour l'administration de cytotoxiques conjugués ou de radioéléments couplés à un anticorps spécifique. À titre d'exemple, des essais in vivo de protéines chimériques associant Fab humain anti- $\alpha v \beta 3$ et interleukine-12 ont été menés chez des souris porteuses de différents types de tumeurs [26]. L'utilisation de ces protéines de fusion a permis d'améliorer l'efficacité antitumorale et antiangiogénique de l'interleukine-12. D'autres travaux ont également fourni des résultats encourageants avec des anticorps couplés à l'interleukine-2, -18, au tumor necrosis factor $\alpha$ (TNF), au tumor necrosis factor-related apoptosis inducing ligand (TRAIL) et à l'interféron gamma.

\section{Conclusion}

La plupart des travaux sur l'inhibition de l'angiogenèse visaient à mettre au point de nouveaux traitements du cancer. Cependant, le traitement de nombreuses pathologies non tumorales au cours desquelles l'angiogenèse joue un rôle pathogénique pourrait directement en bénéficier. En ophtalmologie, l'efficacité du ranibizumab (Lucentis ${ }^{\circledR}$ ), fragment Fab humanisé anti-VEGF, a été démontrée dans le traitement de la dégénérescence maculaire liée à l'âge [14] dont il est maintenant devenu un standard du traitement $(\rightarrow)$. Le bévacizumab a également été testé dans quelques indications ophtalmologiques dans lesquelles il a donné de bons résultats [15]. En rhuma- $(\rightarrow)$ voir N. Leveziel et al., page 1105 tologie, le MEDI-522, Acm anti-intégrine $\alpha v \beta 3$, est testé dans la polyarthrite rhumatoïde [16] et d'autres Acm ciblant l'angiogenèse pourraient l'être bientôt. Enfin, des essais préliminaires d’Acm utilisés pour cibler les compartiments vasculaires, de façon directe ou indirecte, sont en cours dans de multiples domaines: maladie de Crohn, prévention de l'obstruction et de l'hyperplasie intimale après pose de stents vasculaires, etc.

La validation de la stratégie de ciblage vasculaire dans le traitement de différents cancers, confortée par le succès du bévacizumab en pratique clinique, ouvre la voie au développement de nouveaux Acm anti angiogéniques en cancérologie. Au-delà de la cancérologie, l'efficacité de ces biomédicaments pourrait concerner bien d'autres domaines pathologiques et des travaux précliniques et cliniques sont en cours. Le nombre d'Acm thérapeutiques utilisés à des fins de ciblage vasculaire et leurs indications pourraient bien se multiplier dans les années à venir... I'histoire ne fait que commencer. $\diamond$

\section{SUMMARY}

Tumour angiogenesis inhibition

by therapeutic monoclonal antibodies

The development of therapeutic monoclonal antibodies ( $m A b)$ provided many great advances in the treatment of some diseases, in particular cancers. In the treatment of cancers, tumour angiogenesis inhibition by mAb occupies a major place. Tumour angiogenesis is a highly complex multifactor process in which many molecules, which originate both in the tumor cells or their environment, display pro- or anti-angiogenic functions. Many molecular pathways, among which those triggered by vascular endothelial growth factor, platelet-derived growth factor and integrins, are currently under investigation in therapeutic anti-angiogenesis research. Bevacizumab is to date the only mAb targeting the tumour vasculature that is currently used in clinical practice. Clinical trials have revealed its efficacy in colorectal, breast, kidney and lung cancers and gliobastoma multiforme. Such a success confirms the effectiveness in the treatment of cancer of using mAb inhibiting tumour angiogenesis, and many $\mathrm{mAb}$ targeting different angiogenesis pathways will probably be developed. Beyond clinical oncology, angiogenesis inhibition by $\mathrm{mAb}$ is of increasing interest in many other fields of medicine. It could offer interesting therapeutic strategies in some cardiovascular or rheumatologic diseases and currently represents the standard cure in some blinding ocular disorders. $\diamond$

\section{CONFLIT D'INTÉRÊTS}

Les auteurs déclarent n'avoir aucun conflit d'intérêts concernant les données publiées dans cet article.

\section{RÉFÉRENCES}

1. Folkman J. Tumor angiogenesis : therapeutic implications. N EnglJ Med $1971 ; 285: 1182-6$.

2. Ferrara N. VEGF and the quest for tumour angiogenesis factors. Nat Rev Cancer $2002 ; 2: 795-803$.

3. Pugh CW, Ratcliffe PJ. Regulation of angiogenesis by hypoxia : role of the HIF system. Nat Med $2003 ; 9:$ 677-84.

4. Mattern J, Koomägi R, Volm M. Association of vascular endothelial growth factor expression with intratumoral microvessel density and tumor cell proliferation in human epidermoid lung carcinoma. BrJ Cancer 1996 ; $73: 931-4$

5. Claffey KP, Brown LF, del Aguila LF, et al. Expression of vascular permeability factor/vascular endothelial growth factor by melanoma cells increases tumor growth, angiogenesis, and experimental metastasis. Cancer Res 1996; $56: 172-81$.

6. Hurwitz H, Fehrenbacher L, Novotny W, et al. Bevacizumab plus irinotecan, fluorouracil and leucovorin for metastatic colorectal cancer. $N$ Engl J Med $2004 ; 350: 2335-42$.

7. Sandler A, Gray R, Perry MC, et al. Paclitaxel-carboplatin alone or with bevacizumab for non small cell lung cancer. $N$ Engl J Med 2006 ; $355: 2542-50$.

8. Miller K, Wang M, Gralow J, et al. Paclitaxel plus bevacizumab versus paclitaxel alone for metastatic breast cancer. N Engl J Med 2007 ; $357: 2666-76$. 


\section{RÉFÉRENCES}

9. Rini BI, Halabi S, Rosenberg JE, et al. Bevacizumab plus interferon alfa compared with interferon alfa monotherapy in patients with metastatic renal cell carcinoma: CALGB 90206. J Clin Oncol 2008; $26: 5422-8$.

10. Halimi JM, Azizi M, Bobrie G, et al. Vascular and renal effects of antiangiogenic therapy. Nephrol Ther 2008; 4:602-15.

11. Vredenburgh JJ, Desjardins A, Herndon JE $2^{\text {nd }}$, et al. Bevacizumab plus irinotecan in recurrent glioblastoma multiforme.J Clin Oncol 2007 ; 25 : 4722-9.

12. Hunt S. Technology evaluation: IMC-1Cl1, ImClone systems. Curr Opin Mol Ther 2001 ; $3: 418-24$.

13. Miao HQ, Hu K, Jimenez $X$, et al. Potent neutralization of VEGF biological activities with a fully human antibody Fab fragment directed against VEGF receptor 2. Biochem Biophys Res Commun $2006 ; 345: 438-45$.

14. Rosenfeld PJ, Brown DM, Heier JS, et al. Ranibizumab for neovascular age-related macular degeneration. N EnglJ Med 2006 ; 355 : 1419-31.

15. Spaide RF, Fisher YL. Intravitreal bevacizumab (Avastin ${ }^{\oplus}$ ) treatment of proliferative diabetic retinopathy complicated by vitrous hemorrhage. Retina $2006 ; 26: 275-8$.

16. Lainer DT, Brahn $\varepsilon$. New antiangiogenic strategies for the treatment of proliferative synovitis. Expert Opin Investig Drugs 2005 ; 14 : 1-17.

17. Armulik A, Abramsson A, Betsholtz C. Endothelial/pericyte interactions. Circ Res 2005 ; $97: 512-23$.

18. Shen J, Vil MD, Zhang H, et al. An antibody directed against PDGF receptor beta enhances the anti-tumor and the anti-angiogenic activities of an anti-VEGF receptor 2 antibody. Biochem Biophys Res Commun 2007 ; 357 : 1142-7.

19. Javerzat $S$, Auguste P, Bikfalvi A. The role of Fibroblast Growth Factor in vascular development. Trends Mol Med $2002 ; 8: 483-9$

20. Shimada N, Ishii T, Imada T, et al. A neutralizing anti-fibroblast growth factor 8 monoclonal antibody shows potent antitumor activity against androgen-dependant mouse mammary tumors in vivo. Clin Cancer Res 2005; 11:3897-904.
21. Wikström P, Stattin P, Franck-Lissbrant I, Damber JE, Bergh A.

Transforming growth factor betal is associated with angiogenesis, metastasis, and poor clinical outcome in prostate cancer. Prostate 1998 ; $37: 19-29$.

22. Tuxhorn JA, Mac Alhany SJ, Yag F, et al. Inhibition of transforming growth factor-beta activity decreases angiogenesis in a human prostate cancer-reactive stroma xenograft model. Cancer Res 2002; 62:6021-5.

23. Maisonpierre PC, Suri C, Jones PF, et al. Angiopoietin-2, a natural antagonist for Tie2 that disrupts in vivo angiogenesis. Science 1997 ; $277: 55-60$.

24. Oliner J, Min H, Leal J, et al. Suppression of angiogenesis and tumor growth by selective inhibition of Angiopoietin-2. Cancer Cell 2004; $6: 507-16$.

25. Delbaldo C, Raymond $\varepsilon$, Vera K, et al. Phase I and pharmacokinetic stud of etaracizumab (Abegrin ${ }^{\oplus}$ ), a humanized monoclonal antibody against alphaVbeta 3 integrin receptor, in patients with advanced solid tumors. Invest New Drugs $2008 ; 26: 35-43$.

26. Halin C, Rondini S, Nilsson F, et al. Enhancement of the antitumor activity of Interleukin-12 by targeted delivery to neovasculature. Nat Biotechnol $2002 ; 20: 264-9$.

27. Galaup A, Germain S. Blocking PIGF, a future in anti-angiogenic therapy? Med Sci (Paris) $2008 ; 24:$ 459-62.

28. Escudier B, Gross-Goupil M. Revolution in treatment of renal carcinoma: molecular rational and clinical approach. Med Sci (Paris) 2008 ; 24 : 697-9.

\section{TIRÉS À PART}

N. Cézé

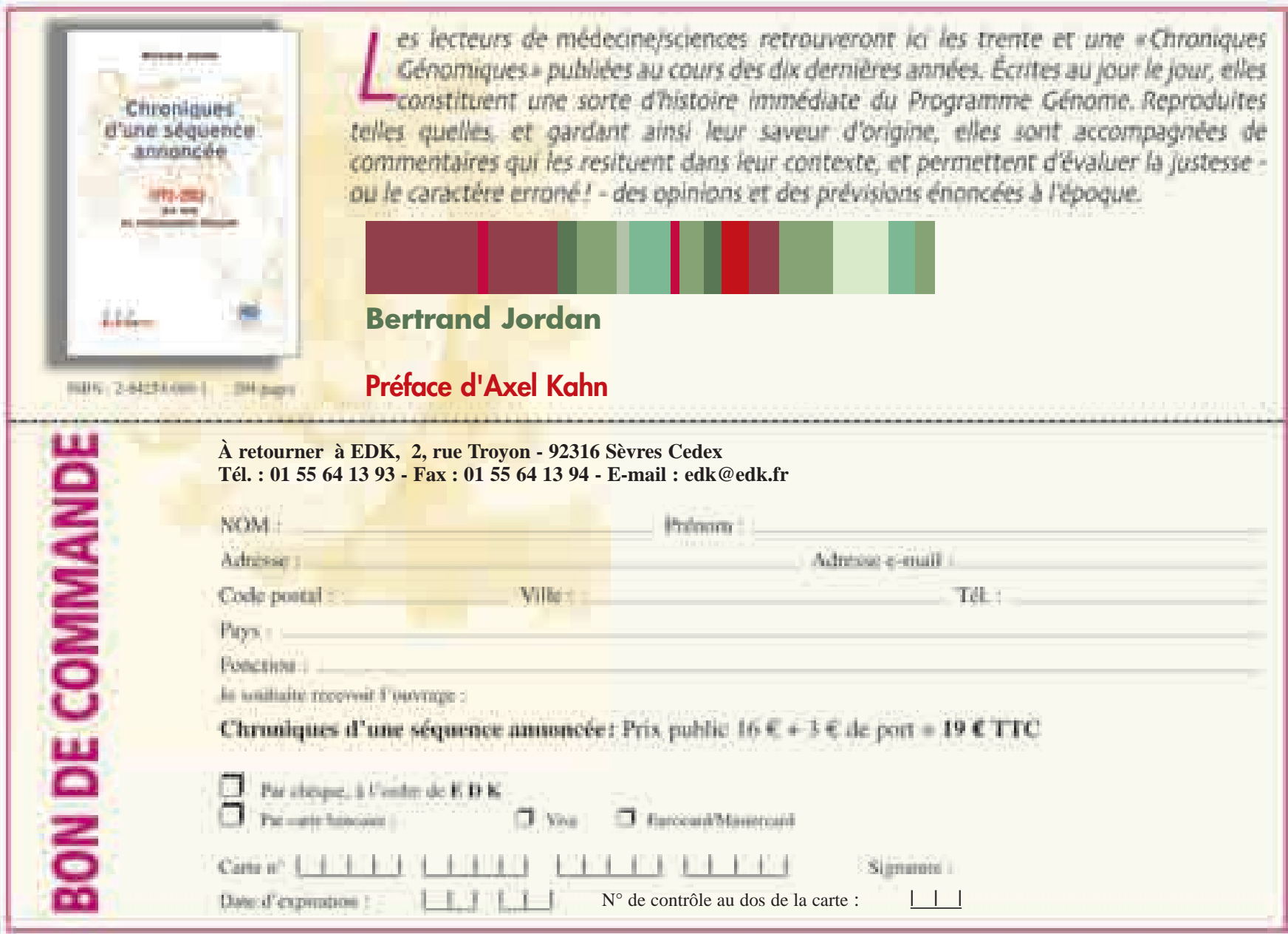

